\title{
Current trends to control fungal pathogens: exploiting our knowledge in the host-pathogen interaction
}

This article was published in the following Dove Press journal: Infection and Drug Resistance

\author{
Laura C García-Carnero' \\ Luis A Pérez-García ${ }^{2}$ \\ José A Martínez-Álvarez' \\ Juana E Reyes-Martínez' \\ Héctor M Mora-Montes' \\ 'Department of Biology, Division \\ of Exact and Natural Sciences, \\ Campus Guanajuato, Universidad de \\ Guanajuato, ${ }^{2}$ Multidisciplinay Academic \\ Unit, Zona Huasteca, Universidad \\ Autónoma de San Luis Potosí, Ciudad \\ Valles, México
}

\begin{abstract}
Human fungal infections remain a major challenge in medicine. Only a limited number of antifungal drugs are available, which are often related to severe adverse effects. In addition, there is an increased emergence related to resistant strains, which makes imperative to understand the host-pathogen interactions as well as to develop alternative treatments. Host innate and adaptive immunity play a crucial role controlling fungal infections; therefore, vaccines are a viable tool to prevent and treat fungal pathogens. Innate immunity is triggered by the interaction between the cell surface pattern recognition receptors (PRRs) and the pathogenassociated molecular patterns (PAMPs). Such an initial immunological response is yet little understood in fungal infections, in part due to the complexity and plasticity of the fungal cell walls. Described host cell-fungus interactions and antigenic molecules are addressed in this paper. Furthermore, antigens found in the cell wall and capsule, including peptides, glycoproteins, glycolipids, and glycans, have been used to trigger specific immune responses, and an increased production of antibodies has been observed when attached to immunogenic molecules. The recent biotechnological advances have allowed the development of vaccines against viral and bacterial pathogens with positive results; therefore, this technology has been applied to develop anti-fungal vaccines. Passive immunization has also emerged as an appealing alternative to treat disseminated mycosis, especially in immunocompromised patients. Those approaches have a long way to be seen in clinical cases. However, all studies discussed here open the possibility to have access to new therapies to be applied alone or in combination with current antifungal drugs. Herein, the state of the art of fungal vaccine developments is discussed in this review, highlighting new advances against Candida albicans, Aspergillus fumigatus, Cryptococcus neoformans, Paracoccidioides brasiliensis, and Sporothrix spp.
\end{abstract}

Keywords: anti-fungal immunity, candidiasis, aspergillosis, cryptococcosis, paracoccidioidomycosis, sporotrichosis

\section{Introduction}

For a long time, innate immunity was considered as a redundant and dispensable line of defense; however, it is now known that despite the lack of specificity, like that of the adaptive immunity, it can distinguish self- from non-self-elements and can activate adaptive mechanisms by the provision of specific signals. When interacting with fungal cells, the elements of the immune system are forced to face unique challenges, by recognizing conserved molecular structures on the pathogen surface, known as pathogen-associated molecular patterns (PAMPs), via conserved transmembrane or soluble receptors, named pattern recognition receptors (PRRs). The
Correspondence: Héctor $M$

Mora-Montes

Departamento de Biología, División de

Ciencias Naturales y Exactas, Campus

Guanajuato, Universidad de Guanajuato,

Noria Alta s/n, col. Noria Alta, C.P.;

Guanajuato Gto. 36050, México

Tel +52 4737320006 Ext 8193

Email hmora@ugto.mx 
best-defined receptors involved in fungal recognition are the Toll-like receptors (TLRs) and the C-type lectin receptors (CLRs). ${ }^{1}$ Upon engagement of PRRs with PAMPs, immune cells produce humoral mediators (inflammatory cytokines, chemokines, or complement elements) or uptake the fungal cell, to eliminate the pathogen and to initiate the adaptive responses. ${ }^{1-3}$ Epithelial cells, neutrophils, macrophages, monocytes, and dendritic cells (DCs) are the main innate immune cells in establishing a protective immune response. ${ }^{2,3}$

The most important and better characterized TLRs participating in fungal recognition are TLR2 and TLR4. Several studies have demonstrated that TLR2 activation tilts the host immunity toward an anti-inflammatory Th2-based response, with the induction of macrophage-deactivating cytokines, such as interleukin-10 (IL-10). On the contrary, activation of TLR4 induces a Th1-based response, with the secretion of pro-inflammatory cytokines. ${ }^{2,4}$ Nevertheless, it has been demonstrated that TLR2 can produce a pro-inflammatory response, although weaker than that mediated by TLR $4 .{ }^{5}$ One of the first evidence suggesting a key role of these receptors in the establishment of a proper antifungal response comes from the study of the Candida albicans-immune cell interaction. ${ }^{6}$ Mice with a deficient TLR4 gene had an impaired recruitment of neutrophils to the infection site and an increased probability to develop disseminated candidiasis. These defects were mediated by a decrease in the release of the keratinocytederived chemokine and the macrophage inflammatory protein $2{ }^{6}$ It has also been demonstrated that genetic variations in TLR1 are associated with an increased risk of candidiasis. ${ }^{7}$ Another important role of TLRs in the antifungal immune response comes from the study of immunity against Aspergillus. Both TLR2 and TLR4 are able to recognize conidia and hyphae of Aspergillus fumigatus and Aspergillus niger and are crucial for neutrophil stimulation during infection. A. fumigatus conidia are recognized by TLR4 and TLR2, inducing the production of pro-inflammatory cytokines, while hyphae are recognized only by TLR2, resulting in the secretion of IL- $10 .^{2}$ In addition, the pro-inflammatory effect of TLR4 has been shown to be protective against invasive aspergillosis, as data show an increased susceptibility to infection in $t l r 4^{-1-}$ mice. ${ }^{4,5}$ Additional studies have demonstrated that a better recognition of $A$. fumigatus needs germination of conidia. Resting conidia are unable to induce cytokine production by macrophages, but swollen conidia and germ tubes can be recognized by Dectin- 1 and TLR $2 .{ }^{8}$

It is now well-established that engagement of TLR2 and TLR4, and activation of the MyD88-dependent signaling pathway, plays a pivotal role in cytokine secretion and activation of the phagocytic process, ${ }^{2}$ but they are insufficient to promote a protective immune response, suggesting the participation of more components during the recognition of fungal pathogens.

The cell wall and the capsule are essential structures in the fungus-host interaction, since they are the first points of contact with the host surfaces, and several fungal polysaccharides have been identified as PAMPs recognized by PRRs, such as the CLRs. ${ }^{1,9}$ These lectins are transmembrane proteins that control signaling processes, microbicidal activity, and phagocytosis and are involved directly in the host innate response, since fungal cell surfaces are carbohydrate-rich structures. $^{2}$ One of these receptors, Dectin-1, is a primary non-opsonic receptor for phagocytosis of soluble and particulate $\beta 1,3$-glucan and can contribute to the recognition of this carbohydrate already opsonized. ${ }^{4,7}$ Dectin- 1 is able to mediate specific recognition of $\beta 1,3$-glucan found in the cell walls of several fungi such as Candida, Aspergillus, Pneumocystis, Sporothrix, and Coccidioides. ${ }^{1,10,11}$ In some cases, Dectin-1 and TLR2 can collaborate to increase pro-inflammatory cytokine production. ${ }^{4}$ Fungal recognition by Dectin-1 depends on the exposure of $\beta 1,3$-glucan at the surface of the wall, which can vary among different types of fungi and different morphologies. ${ }^{10}$ Dormant Aspergillus conidia are not able to activate Dectin-1, given that this morphology does not express $\beta 1,3$-glucan on the cell surface. After germination, $\beta 1,3$-glucan is exposed and can be detected by Dectin- 1 . $^{7}$ Although mannans can function as immune evaders, several host receptors are able to recognize them and enhance host immunity. ${ }^{12}$

Dectin-2 and Dectin-3 recognize $\alpha$-mannan and can form heterodimeric structures that provide high affinity to the binding of mannans to activate intracellular signaling cascades. ${ }^{12,13}$ Dectin-2 lacks intracellular signaling motifs and therefore has to associate with other receptors to be able to transduce signals. ${ }^{8,12}$ All three Dectins are responsible for the induction of a Th17 response, a key response in defense against fungal pathogens. ${ }^{13}$ The macrophage inducible C-type lectin (Mincle) is a type II transmembrane receptor that binds $\alpha$-mannosyl residues but not mannans, indicating recognition of terminal $\alpha$-mannoses. ${ }^{13}$ It has been demonstrated that Mincle binds to C. albicans and Saccharomyces cerevisiae but also recognizes Malassezia spp. In experimental models of $C$. albicans infection, mice lacking Mincle expression showed higher fungal loads in kidneys and impaired production of tumor necrosis factor- $\alpha$ (TNF $\alpha$ ) by macrophages. ${ }^{7,8,13}$ Mannose receptor (MR) is a type I transmembrane protein that recognizes mannose, fucose, and $\mathrm{N}$-acetylglucosamine 
residues and can be expressed on the cell surface and as a soluble form released during proteolytic cleavage. ${ }^{8,12,13}$ It has an important role during phagocytosis, due to not only particle binding but also signaling by its intracellular portion able to couple detection of particles to phagocytosis activation. ${ }^{13}$ In macrophages, it recognizes fungal mannans and mediates recognition and phagocytosis of Candida spp. ${ }^{14}$ In addition, DCs can internalize and process mannoproteins through this receptor, leading to maturation and activation. ${ }^{12}$ In collaboration with TLR9 and NOD2, MR can recognize chitin particles, leading to the production of IL-10 and establishing an anti-inflammatory process. ${ }^{15}$ However, it has been demonstrated that MR can be dispensable for immunity to several fungi, given that mannans can be recognized by other receptors, such as Dectin- 2 and TLR $4 .{ }^{8}$ The DC-specific intercellular adhesion molecule 3-grabbing nonintegrin (DC-SIGN) receptor can bind $N$-linked mannans and mannose-containing oligosaccharides. It functions as an endocytic receptor and is expressed on professional presenting cells. ${ }^{12}$ This receptor, as well as MR, is activated primarily by IL-4 and is associated with a Th2 response. ${ }^{2}$ It has been found to participate in the phagocytosis of Candida and Aspergillus spp. ${ }^{5}$

Recently, the melanin-sensing CLR (MelLec) has been identified. This protein interacts with the naphthalene-diol unit of 1,8-dihydroxynaphthalene-melanin found on the surface of conidia from $A$. fumigatus and other melanized fungi. MelLec is found on the surface of endothelial and myeloid cells and is required for the protection against disseminated aspergillosis. ${ }^{16}$

\section{The immunogenic ability of fungal components}

During the recognition of a fungal pathogen, macrophages and neutrophils play a key role. Upon PRR-PAMP interaction, downstream signaling pathways trigger the induction of antimicrobial mechanisms and the release of proinflammatory cytokines, to stimulate and attract other leukocytes to the site of infection. ${ }^{15,17}$ IL-10 and IL-17 are key cytokines for the defense against fungi. IL-17 is in charge of stimulating granulopoiesis and neutrophil recruitment, essential for the Th17 response. Fungal cell wall components, such as $C$. albicans mannans and $\beta$-glucans, induce prostaglandin $\mathrm{E} 2$, an important proinflammatory mediator for the Th17 response, via MR and Dectin-1, respectively. On the contrary, IL-10 is a Th2-derived cytokine that shifts the balance toward antiinflammatory responses. Increased IL-10 production exacerbates Candida infection in mice, due to a downregulated Th1 antifungal response. ${ }^{1}$
In fungal cells, sphingolipids have several roles in signal transduction, cell cycle, apoptosis, and pathogenesis. C. neoformans and other fungal pathogens are able to produce antigenic glycosphingolipids (GSLs), such as glucosylceramide (GlcCer). The immunogenicity and clinical significance of these GSLs have been demonstrated in several fungal infections, such as cryptococcosis, aspergillosis, histoplasmosis, paracoccidioidomycosis, and chromoblastomycosis. Patients with these mycoses show high levels of immunoglobulin $\mathrm{G}$ (IgG1) IgG1 to GlcCer, and these antibodies are able to inhibit Cryptococcus growth and budding. ${ }^{18}$

Vesicles have been recognized as important structures related to pathogen virulence and modulation of the host immunity. Exosome-like vesicles containing virulence factors and antigens have been characterized in fungi, such as C. neoformans. These vesicles can carry ergosterol, GlcCer, and glucuronoxylomannan (GXM). GXM is the Cryptococcus major capsular polysaccharide and participates in the pathogenesis of the fungus, by diminishing NO production and increasing IL-10 and TGF- $\beta$ secretion as part of a depressive effect on the immune system. ${ }^{1}$

Little is known about specific antigens in A. fumigatus that are able to induce an antifungal T-cell response. A positive T-cell response toward some Aspergillus antigens was seen with an analysis of 22 patients with invasive aspergillosis. Pep1, Crf1, Gel1, Sod1, $\alpha 1,3$-glucan, and $\beta 1$,3-glucan correlated with a good disease outcome, indicating the existence of protective epitopes within these proteins. Nevertheless, only one specific epitope has been reliably used to expand specific $\mathrm{T}$ cells in vitro, the p41 peptide found within the Crfl protein. ${ }^{19}$

Della Terra et al developed a subcutaneous murine model of sporotrichosis, mimicking the horizontal and zoonotic transmission of the mycosis. They analyzed the protein secretion profiles of 11 isolates of Sporothrix brasiliensis and saw that $\mathrm{IgG}$ antibodies produced by infected mice recognized 17 proteins ranging from 22 to $130 \mathrm{kDa}$ in the whole cell extract and 38 to $100 \mathrm{kDa}$ in the exoantigens. There were two immunodominant molecules detected in almost $100 \%$ of the cases, Gp70 and a $100 \mathrm{kDa}$ protein. A high level of cross-reaction between Sporothrix schenckii and $S$. brasiliensis antigens was detected, supporting the idea that the epitopes are conserved in different species of the clinical clade. ${ }^{20}$

Several fungal pathogens, such as Fonsecaea pedrosoi, Paracoccidioides brasiliensis, A. fumigatus, C. neoformans, and $S$. schenckii, express sialic acids on the cell surface. Sialic acids have important role in fungal pathogenesis, 
participating in adhesion to host surfaces and protection from the immune response. Sialic acid-depleted cells from $C$. neoformans and $S$. schenckii are more susceptible to uptake by phagocytic cells, while removal of this sugar from F. pedrosoi surface increased the ability of these cells to interact with neutrophils. ${ }^{21}$ On the contrary, when sialic acids are removed from A. fumigatus conidia, the uptake by pneumocytes and macrophages is reduced. ${ }^{22}$

From this information, it is clear that fungal elements are recognized by the host immunity, and some of them can be associated with a protective immune response. This has stimulated the field to search for antigens that can be used to develop vaccines against fungal pathogens. In the next sections, we summarize the current knowledge in this field.

\section{Vaccine developments against C. albicans}

Due to the increased drug resistance in fungi, it is necessary to find alternatives to fight against fungal diseases. CRM45 and CRM197, two diphtheria toxin-related proteins, named cross-reacting materials (CRMs), have attracted the attention in studying the properties of chimeric toxins or new vaccine development. ${ }^{23}$ A glycoconjugate (Lm-CRM197) composed of $\beta 1,3$-glucan polysaccharide and diphtheria toxoid CRM197 has been used as a preventive vaccine against both systemic and vaginal candidiasis in mice and rats, respectively. The protein coupled to laminarin converts this polysaccharide to immunogenic, which induces anti- $\beta$-glucan antibodies in both mice and rats. The antibodies, mostly of the IgG class, were essential for anti-Candida protection. ${ }^{24}$ The first attempt to develop a Candida-derived ribosomal vaccine was proposed by Levy et al, where $C$. albicans ribosomal fractions containing both RNA and protein were injected subcutaneously in mice. Then, the animals were challenged either intraperitoneally or intravenously (IV) with live yeast, and 30 days after the challenge, those mice that received the ribosomal fraction increased their survival from $46 \%$ to $90 \%$ compared with $14 \%$ to $38 \%$ of controls. Those mice immunized with the ribosomal fraction and IV challenged had a survival rate of $22 \%-67 \%$, whereas none of the unimmunized mice survived. ${ }^{25}$ Another immunogenic determinant of the $C$. albicans cell wall is the phosphomannan complex (PMC). Vaccinated mice with liposome-encapsulated PMC developed longer survival time when were challenged with the fungal yeast than control groups. Likewise, if PMC is coupled with BSA, the protection against $C$. albicans is enhanced in this model of infection. ${ }^{26,27}$ Furthermore, mice vaccinated subcutaneously with $C$. albicans-derived mannan or mannan-BSA conjugate showed a mannan dose-dependent induced protection, improved 40 -fold by the conjugation of BSA to the glycan. ${ }^{28}$

The cell wall components of $C$. albicans such as proteins are crucial for virulence and pathogenicity. Such is the case of Als3, a hyphal-specific Glycosylphosphatidylinositolanchored cell wall protein and a member of the C. albicans agglutinin-like sequence family. These proteins have several biological functions, namely cell growth, biofilm formation, cell adhesion, host invasion, and iron acquisition. ${ }^{29}$ Thus, Als3 has become an important target to design vaccines and antibodies to control candidiasis. The mice vaccinated with rAls $3 \mathrm{p}-\mathrm{N}$, a vaccine produced in $S$. cerevisiae using the N-terminal domain of Als3, showed $50 \%$ reduced mortality after the challenge with $C$. albicans compared with $100 \%$ of lethality on the control group. ${ }^{30}$ Another safe vaccine proved against the same fungus is NDV-3, which contains Als3p-N plus a six-His tag. A significant feature of this vaccine is the capacity to cause memory B- and T-cell immune responses. The efficacy of this vaccine on vulvovaginal candidiasis model depends on the participation of both B and T cells. ${ }^{31,32}$ Meanwhile, protective antibodies associated with Als3p have been developed to protect against $C$. albicans. The 3-A5 and 113 are monoclonal antibodies (MAbs) produced in Pichia pastoris and $S$. cerevisiae, respectively. The first MAb 3-A5 was against a fragment representing amino acids 18-329 of the N-terminus of Als3, while MAb 113 was against the N-terminal 433 amino acids of the same protein. Both antibodies are able to bind specifically to the hyphae, but not yeast cells, and avoid $C$. albicans adhesion to vascular endothelial cells and buccal epithelial cells. ${ }^{33}$ Another MAb, C7, was produced in BALB/c mice by intraperitoneal injection of C. albicans high-molecular-weight stress mannoprotein recognized by salivary secretory immunoglobulin $\mathrm{A}$. MAb C7 is able to recognize $>200 \mathrm{kDa}$ mannoprotein as epitope. In addition, MAb $\mathrm{C} 7$ interferes with the $C$. albicans iron uptake pathway. ${ }^{34-36}$ The MAb 3D9.3 was prepared using a specific fraction of germ tube of $C$. albicans and immunizing BALB/c mice. This antibody is able to avoid C. albicans adhesion to both human buccal epithelial and vascular endothelial cells. Moreover, MAb 3D9.3 can discriminate between $C$. albicans and Candida dubliniensis. With this feature, this antibody can also be used as a diagnostic tool to differentiate between both species. ${ }^{37}$ The cell wall proteins Als3 and Hyr1 were recognized by MAb 2G8, an anti- $\beta$-glucan antibody IgG2b. Using MAb 2 G8 in human epithelial Hep-2 cells, the adherence of hyphae to host cells 
decreased by $45 \%$ compared with controls, and the fungal burden in both vagina and kidney were also reduced. It is noteworthy that this MAb recognized not only $C$. albicans but also $A$. fumigatus and $C$. neoformans ${ }^{38} \mathrm{~A}$ recombinant human antibody, scFv3, is a single-chain variable fragment specific for $C$. albicans Als3. scFv3 can avoid adherence to either epithelial or endothelial human cells. ${ }^{39}$ During the infection process, C. albicans express several proteins, and from them, hyphal wall protein-1 (Hwp1), enolase (Eno1), phosphoglycerate kinase (Pgk1), glyceraldehyde3-phosphate dehydrogenase (Gap1), fructose-bisphosphate aldolase (Fba), and methyltetrahydropteroyltriglutamate (Met6) are highly produced during pathogenesis. ${ }^{40}$ Peptides closer to the N-terminal end of these proteins were conjugated to a $\beta$-mannan trisaccharide epitope. All the glycopeptides, except $\beta$-(Man) $)_{3}$-Pgk1, protected the mice against candidiasis; some of them even confer $80 \%-100 \%$ survival throughout a 120-day post-challenge observation period. In addition, the fungal burden on kidneys was significantly reduced or not found. ${ }^{41}$

\section{Vaccine developments against A. fumigatus}

Aspergillus spp. is one of the main fungal pathogens responsible for the increased number of deep-seated mycosis in immunocompromised populations. Since glycans are the main components of the fungal cell wall, and they are absent in the host cells, an obvious approach for immunization was to develop a glycoconjugate vaccine, as mentioned earlier. One of the first approaches generated was Lm-CRM197 that showed protection against $C$. albicans and A. fumigatus. ${ }^{24}$ In a similar manner, purified cell wall $\alpha$ - and $\beta 1,3$-glucans have been used as immunogens, and protection has been observed after intranasal vaccination. ${ }^{42}$ From a different point of view, development of MAbs seems to be a suitable strategy for antifungal therapy, as already demonstrated for C. albicans. The in vivo protective efficacy of the MAb A9, an IgG1 directed against a peptide antigen found in hyphae and swollen conidia of $A$. fumigatus cell wall, was evaluated in a murine model of invasive aspergillosis with enhanced survival times. ${ }^{43}$

It has been demonstrated that subcutaneous immunization of corticosteroid-immunosuppressed mice using recombinant fungal allergen Asp $\mathrm{f} 3$ was protective against invasive pulmonary aspergillosis, by probably inducing a cellular immune response that leads to the activation of lymphocytic cells upon infection, thus restoring corticosteroid-suppressed phagocytes to clear fungal cells. ${ }^{44}$ In addition, a wide set of recombinant proteins including Pep1, Crf1, and Gell are able to activate Th-cell responses in mice and humans. ${ }^{42}$

Another approach for immunization against aspergillosis has been the use of the inactivated whole fungal cell, such as heat-killed $S$. cerevisiae yeasts, which has been proven to be protective when administered subcutaneously to mice 2 weeks prior to the intravenous infection with A. fumigatus. The protection was potentiated by using alum as an adjuvant. ${ }^{45}$ Previously, it had been already demonstrated that live or heat-killed A. fumigatus cells, as well as crude culture filtrates intranasally inhaled, were able to develop local and peripheral protective Th1 responses in a mouse model of pulmonary aspergillosis, mainly mediated by antigen-specific $\mathrm{CD}^{+} \mathrm{T}$ cells proficient to confer defense upon adoptive transfer to naïve recipients. Interestingly, after drug-induced immunosuppression, only live $A$. fumigatus or the crude filtrate, but not heat-killed fungal cells, achieved prolonged survival in mice. ${ }^{46} \mathrm{~A}$ successice study proved that mice immunized with live conidia induced interferon- $\gamma\left(\right.$ IFN- $\gamma$ ) producing fungus-specific $\mathrm{CD}^{+} \mathrm{T}$ cells, unlike heat-killed conidia that mainly induced $A$. fumigatus-specific $\mathrm{CD}^{+} \mathrm{T}$ cells producing greater amounts of IL-4 and IL-13. ${ }^{47}$

\section{Vaccine developments against C. neoformans}

C. neoformans is a ubiquitous fungal pathogen mainly infecting immunocompromised individuals. ${ }^{48}$ The first described vaccine to confer antibody-mediated protection against a systemic mycosis in an animal model was a conjugate vaccine of GXM, a major component of the cryptococcal capsule, coupled to tetanus toxoid. The vaccine was given to mice with monophosphoryl lipid A as an adjuvant, eliciting high levels of IgA and IgG against the capsular GXM. This immunization conferred $70 \%-80 \%$ protection against an intravenous moderate challenge with $C$. neoformans. The authors also suggested that IGs derived from high-tittered antiserum induced by this vaccine may be used as an immunotherapy for the management of cryptococcosis in patients with AIDS. ${ }^{49}$ Nonetheless, the efficacy of the antibodies elicited against GXM-tetanus toxoid relies on the integrity of the whole immune system, since such efficacy seems to depend on T-cell function and its related cytokines, ${ }^{50,51}$ the antibody isotype ${ }^{52,53}$ and, more remarkably, the host genetics. ${ }^{54}$ Likewise, using P13, a mimotope of $\mathrm{GXM}^{55}$ linked to either tetanus toxoid or BSA that was preadsorbed to the aluminum hydroxide adjuvant Alhydrogel also induced a protective response against the fungus, prolonging survival 
when compared to controls after administration of a lethal challenge ${ }^{56}$ or after the establishment of a chronic infection, though the degree of protection was dependent on the carrier protein, route of infection, and also the genetic background of the host. ${ }^{57}$

On the contrary, alkaline extracts from mutant cryptococcal strains lacking capsule or chitosan were packaged into glucan particles, obtained from purified $S$. cerevisiae cell walls. These preparations were used for subcutaneous vaccination, providing significant protection against pulmonary infection with highly virulent strains of $C$. neoformans and Cryptococcus gattii. ${ }^{58}$ Cytosolic proteins of the pathogen have also been used as an antigen. Khan et al described that poly-lactide co-glycolide microspheres encapsulated with cytosolic proteins further co-encapsulated into fibrin cross-linked plasma beads prompted an effective immune response by activating both $\mathrm{CD}^{+}$and $\mathrm{CD}^{+} \mathrm{T}$ cells, favoring IFN- $\gamma$ - and IL-2-producing cells. Vaccination with this system also induced an IgG response, effectively cleared the fungal burden in vital organs, and increased the survival rate of immunized mice. ${ }^{59}$

An interesting approach to the development of strategies to confer immunity to fungal infections is the one explored by Wormley et al. ${ }^{60}$ In this study, the authors genetically engineered a strain of C. neoformans to produce IFN- $\gamma$, and after the administration of this modified strain, mice were able to resolve the primary infection mainly by stimulation of local Th1-type anti-cryptococcal cell-mediated immune response and demonstrated full protection against a secondary pulmonary challenge with a pathogenic $C$. neoformans strain. ${ }^{60}$ More recently, the same group explored the effect of this vaccination in mice with defective T-cell-mediated immune response. This was achieved by inactivating $\mathrm{CD}^{+}$ or $\mathrm{CD}^{+} \mathrm{T}$ cells with anti-CD4 ${ }^{+}$or anti-CD8 ${ }^{+}$antibodies, respectively, prior to infection with the IFN- $\gamma$-producing $C$. neoformans strain. They found that the absence of $\mathrm{CD}^{+}$or $\mathrm{CD}^{+} \mathrm{T}$ cells, but not both subsets, resulted in survival to an acute pulmonary infection with the engineered strain and a subsequent second challenge with wild-type $C$. neoformans strain. ${ }^{61}$

Considering that antibodies stimulated by vaccination could defend against fungal infections, some investigations have been directed toward the generation of therapeutic MAbs. For example, it has been demonstrated not only that MAbs against melanin reduced the growth rate of in vitro melanized $C$. neoformans cells but also that passive immunization using these MAbs was able to extend the survival of mice infected with this fungus, suggesting the protection of the MAbs to melanized $C$. neoformans could alter the properties of the cell wall melanin, thus interfering with cell replication. ${ }^{62}$ Similarly, MAbs binding to $C$. neoformans capsule directly modified gene expression, and these effects correlated with antibodies binding to different locations of the capsule, ranging from downregulation of genes encoding for protein translation to differences in the pattern of phosphorylated proteins and changes in lipid metabolism that resulted in increased susceptibility to amphotericin B. ${ }^{63}$ Casadevall et al developed 18B7, a murine MAb, against $C$. neoformans polysaccharide that showed interaction with all serotypes of the fungus, activation of the complement system, and promotion of phagocytosis. ${ }^{64}$ More effective results have been accomplished by using this antibody conjugated to ${ }^{213} \mathrm{Bi}$, a therapeutic radioisotope, against cryptococcosis in mice. ${ }^{65}$

\section{Vaccine developments against $P$. brasiliensis}

P. brasiliensis is a dimorphic fungal pathogen causing infection by inhalation of fungal conidia. Paracoccidioidomycosis is the most prevalent subcutaneous mycosis in Latin American countries, especially in Brazil. ${ }^{66}$ Puccia et al reported that gp43 is a specific antigenic and exocellular component of $P$. brasiliensis found in culture filtrates ${ }^{67}$ and ever since has been systematically studied as a potential vaccine. The T-cell epitope of gp43 is a 15-mer peptide (P10), and immunization of mice with both gp43 and P10 directed to strong protection against intratracheal challenge by virulent $P$. brasiliensis. The cellular immune response in mice to gp43 involves $\mathrm{CD}^{+}$ Th1 cells producing IFN- $\gamma$ and IL-2, unlike P10 in which the protective effect is mainly due to an IFN- $\gamma$-mediated cellular immune response and does not induce a humoral response. ${ }^{68}$

Since management of paracoccidioidomycosis often includes extended periods of chemotherapy, a combination of antifungal drugs and immunization with P10 were used together attempting to improve the treatment of the disease and to avoid relapses. This combination, in general, increased the levels of IFN- $\gamma$ and IL-12 as proved to be a successful additive protective effect in mice after intratracheal challenge, ${ }^{69}$ and similar results were obtained with this combined drug/P10 treatment in anergic mice ${ }^{70}$ or using a DNA-based vaccination strategy with the gp43 gene. $^{71}$

A few other antigenic molecules of $P$. brasiliensis have also been investigated, such as paracoccin and the $\mathrm{Pb} 27$ protein. Paracoccin, a dual-function protein having lectin properties as well as $\mathrm{N}$-acetylglucosaminidase activities, is able to stimulate murine peritoneal macrophages to produce TNF $\alpha$ and nitric oxide and also induce Th1 immunity in mice 
when injected prior to fungal challenge. ${ }^{72}$ Immunization with the $\mathrm{Pb} 27$ protein, in addition to its protective effect, also proved to prevent pulmonary fibrosis in mice. ${ }^{73}$

\section{Vaccine developments against Sporothrix spp.}

Species belonging to the genus Sporothrix can be considered worldwide distributed, but endemic areas of the caused disease, sporotrichosis, are found in Latin America and Asia. ${ }^{74}$ S. brasiliensis, S. schenckii, and Sporothrix globosa can cause lymphocutaneous or systemic fungal infections in healthy and immunocompromised hosts. ${ }^{75,76} \mathrm{~S}$. brasiliensis has been recognized as the most virulent strain and represents a threat to humans due to the massive zoonotic transmission in Brazil. ${ }^{77}$

The cell wall of Sporothrix represents a suitable source of antigens, and one is a glycoprotein of $70 \mathrm{kDa}(\mathrm{Gp} 70)$ found in $S$. schenckii and S. brasiliensis. ${ }^{75,78} \mathrm{Gp} 70$ has been shown to be the immunodominant molecule of the Sporothrix cell wall. ${ }^{75}$ Mice infected with Sporothrix are able to produce specific antibodies, such as IgG1 and IgG3 against Gp70, indicating its possible participation in controlling the infection. ${ }^{79}$ Nascimento et al generated a MAb against Gp70, MAb P6E7, of the IgG1 isotype. ${ }^{78}$ Yeast cells opsonized with MAb P6E7 had an increased phagocytic index and a reduction in the number of colony forming units in liver and spleen. Also, MAb P6E7 was able to inhibit the interaction of yeasts with the subendothelial matrix, given that this antigen is a putative adhesin for fibronectin and laminarin. ${ }^{80,81}$ In addition, when the MAb was injected after infection, high levels of IFN- $\gamma$ were detected, indicating that treatment with P6E7 may induce a protective cell-mediated response. ${ }^{79}$ The passive immunization demonstrated to be protective before, during, and after infection, and even in mice infected with highly virulent strains. ${ }^{82}$ Passive immunization with antibodies antiGp70 provided protection against sporotrichosis but was not able to induce the generation of long-lasting memory. These antibodies can be used as a treatment option, especially for immunocompromised patients, but not as stimulators of an active immunity. ${ }^{76}$

Portuondo et $\mathrm{al}^{83}$ developed two vaccine formulation by using glycoproteins from the cell wall of Sporothrix, with different concentrations of proteins (AH-CWP100 and AH-CWP10). Sera from both AH-CWP100- and AHCWP10-immunized mice enhanced yeast phagocytosis and inhibited its adhesion to fibroblast. In addition, IgG1 and IgG2 antibodies were induced by immunization with AHCWP100, and passive transference of this serum was able to provide in vitro protection. Also, there was an increased ex vivo release of IL-12, IFN- $\gamma$, IL-4, and IL-17, suggesting a balance of Th1, Th2, and Th17 responses. ${ }^{83}$ Later, a PGACWP100 vaccine induced higher levels of IgG2, higher phagocytic index of yeast, but similar fungal load reduction, when compared to the AH-CWP100. The authors proposed that several mechanisms are involved in the protection given by the vaccine formulations that might depend on the adjuvant. ${ }^{84}$ Most recently, Chen et al identified three epitopes from the Gp70 sequence and choose one (kpvqhalltplgldr peptide) to express it on a phage. They observed that immunization with the recombinant phage displaying the peptide could produce antibodies that bind to Gp70, which indicates that the peptide has a similar response to that of the whole Gp70, in the treatment of sporotrichosis. The recombinant phage-induced antibody production protected mice infected with $S$. globosa, showing a reduction of the fungal load, a decrease in the number of inflammatory cells and a higher survival rate $(80 \%)$ of animals. ${ }^{85}$ This could be a potential vaccine candidate against Sporothrix, given that the presence of Gp70 has been confirmed in S. schenckii, S. brasiliensis, and S. globosa.

\section{Vaccine developments against other fungal pathogens}

BAD1 is a surface adhesin and an immunodominant antigen of Blastomyces dermatitidis that generates cell-mediated and humoral responses. ${ }^{86}$ Recombinant BAD1 immunization is able to prolong survival of mice to lethal pulmonary blastomycosis, but most mice succumb to infection, even when IL-12 is used as an adjuvant. Wuthrich et $\mathrm{al}^{87}$ tested a recombinant, live, attenuated vaccine against $B$. dermatitidis, the BAD1 null mutant. The mutant is unable to establish a lethal pulmonary infection since mice infected with it could control the multiplication of yeast and generated acquired immunity. Immunized mice with viable yeasts of the mutant showed a decreased fungal burden in lungs and developed a delayed-type hypersensitivity and polarized type 1 cytokine response, linked with resistance. ${ }^{87}$ This protection was correlated with the production of IFN- $\gamma$ and T-cell activation and proliferation. ${ }^{86}$

In the case of Histoplasma capsulatum, a $62 \mathrm{kDa}$ protein (termed HIS-62) was isolated from yeast cells. It was determined that this antigen is a target of the cellular response and that is able to confer protection against the mycosis. In mice infected intravenously with yeast cells, immunization with the protein induced a high proportion of monoclonal population of $\mathrm{T}$ cells and stimulated a protective immune response. ${ }^{88}$ Also, immunization stimulates the production of 
IL-10, IL-12, and IFN- $\gamma \cdot{ }^{86}$ Later, the recombinant HIS-61 was expressed and tested for immunological activity. Vaccination with this protein caused an increase in the recognition of the yeast by the lymphocytes and a higher survival rate, providing protection against a lethal intranasal challenge with yeast in a mouse model. ${ }^{89}$

Once an infection caused by Coccidioides is resolved, memory immune cells confer lifelong immunity against a re-infection. Several antigens of the fungus have been identified and tested to see whether they might induce a similar protection than that of the natural infection..$^{90}$ Two antigens have recently been tested, a proline-rich cell wall protein, Ag2/PRA, and a secreted protein, Coccidioides-specific antigen (CSA). Protection has been reported for the recombinant protein Ag2/PRA and its truncations, but the CSA has not been implicated in a protective response yet. However, Shubitz et $\mathrm{al}^{91}$ developed a vaccine based on a chimeric fusion protein composed of Ag2/PRA ${ }_{1-106}$ co-expressed with CSA and tested it in a mouse intranasal challenge model. They observed greater survival of mice when immunized with the chimeric protein, compared to either antigen alone. ${ }^{91}$

\section{Passive immunotherapy}

The use of antibodies to neutralize an infection without inducing an active memory immune response by the host is another way to combat pathogens. Mycograb ${ }^{\circledR}$ a human recombinant antibody against Hsp90, a chaperone essential for cell viability in Candida spp., shows antifungal activity and synergy with amphotericin B. Aspergillus spp. and C. neoformans also possess Hsp90; therefore, this antibody could also be used against these pathogens. ${ }^{92}$ The $18 \mathrm{~B} 7$, a mouse MAb directed against $C$. neoformans capsular polysaccharide component, GXM could be used as anti-capsular antibody therapy, promoting opsonophagocytosis of the fungi or clearance GXM. ${ }^{93}$ $H$. capsulatum is another pathogenic fungus that expresses in the cell surface an antigen identified as a histone H2B-like protein, and the administration of a MAb directed against it decreased the fungal burden and pulmonary inflammation and increased the yeast phagocytosis by J774.16 cells through a CR3-dependent process. ${ }^{94}$ MAbs both IgM(B6.1) and $\operatorname{IgG} 3(\mathrm{C} 3.1)$ directed against a $\beta 1,2$-mannotriose epitope of $C$. albicans cell surface have a protective role in a murine model of candidiasis. ${ }^{53}$ The killer toxin, which interacts with $\beta$-glucans, is another option to combat fungal infections through passive immunotherapy. ${ }^{95}$ The anti-idiotypic killer toxin antibodies were produced by immunizing animals with killer toxin. ${ }^{96}$ These antibodies decreased vaginal candidiasis and Pneumocystis carinii pneumonia. ${ }^{95}$

\section{Concluding remarks}

The increased frequency of multidrug resistance in fungal isolates and the emergence of new species naturally resistant to the current chemotherapeutic alternatives, such as Candida auris,${ }^{97}$ have stressed the need for alternatives to control fungal pathogens. The examples given in this review paper underscore the effort in this line, and currently, there are some antigens/antibodies with the potential to confer protection against fungal pathogens tested in clinical trials. ${ }^{23}$ We have to take this information carefully, as fungal organisms have several strategies to evade immune recognition, ${ }^{1}$ and there is a risk of failure in the immunization against the pathogen, as in the case of C. albicans. ${ }^{23}$ Multivalent antigenic preparations might represent an alternative to divert this potential risk, and this is a challenge of high priority to address. Furthermore, the study of universal vaccines, like WGA-Fc, a chimera that binds chitin and has antifungal properties, ${ }^{98}$ may open new alternatives to generating a vaccine that is able to protect against not only one but several fungal pathogens. Finally, the combination of antifungal drugs and immunostimulants, like fungal antigens, is another promising area to develop new therapeutic schemes that need further development.

\section{Acknowledgments}

This work was supported by Consejo Nacional de Ciencia y Tecnología (ref. PDCPN2014-247109, and FC 201502-834), Universidad de Guanajuato (ref. DAIP CIIC 95 / 2018), and Red Temática Glicociencia en Salud (CONACYT-México). LAPG was supported by Programa para el Desarrollo Profesional Docente (ref. UASLPPTC-607) and Universidad Autónoma de San Luis Potosí (ref. C17-FAI-06-12.12).

\section{Disclosure}

The authors reports no conflicts of interest in this work.

\section{References}

1. Hernandez-Chavez MJ, Perez-Garcia LA, Nino-Vega GA, Mora-Montes HM. Fungal strategies to evade the host immune recognition. J Fungi (Basel). 2017;3:51.

2. Calich VL, Pina A, Felonato M, Bernardino S, Costa TA, Loures FV. Toll-like receptors and fungal infections: the role of TLR2, TLR4 and MyD88 in paracoccidioidomycosis. FEMS Immunol Med Microbiol. 2008;53:1-7.

3. Jiang S. Immunity against Fungal Infections. Immunology and Immunogenetics Insights. 2016;8:3-6.

4. Chai LY, Netea MG, Vonk AG, Kullberg BJ. Fungal strategies for overcoming host innate immune response. Med Mycol. 2009;47:227-236.

5. Netea MG, Van der Meer JWM, Kullberg BJ. Role of the dual interaction of fungal pathogens with pattern recognition receptors in the activation and modulation of host defence. Clin Microbiol Infect. 2006;12:404-409. 
6. Netea MG, van der Graaf C, Vonk AG, Verschueren I, Van der Meer JWM, Kullberg BJ. The role of Toll-like receptor (TLR) 2 and TLR4 in the host defense against disseminated candidiasis. $J$ Infect Dis. 2002;185:1483-1489.

7. UnderhiII DM, Pearlman E. Immune interactions with pathogenic and commensal fungi: a two-way street. Immunity. 2015;43:845-858.

8. Figueiredo RT, Carneiro LA, Bozza MT. Fungal surface and innate immune recognition of filamentous fungi. Front Microbiol. 2011;2:248.

9. Díaz-Jiménez DF, Pérez-García LA, Martínez-Álvarez JA, MoraMontes HM. Role of the fungal cell wall in pathogenesis and antifungal resistance. Curr Fungal Infect Rep. 2012;6:275-282.

10. Martinez-Alvarez JA, Perez-Garcia LA, Mellado-Mojica E, et al Sporothrix schenckii sensu stricto and Sporothrix brasiliensis are differentially recognized by human peripheral blood mononuclear cells Front Microbiol. 2017;8:843.

11. Jellmayer JA, Ferreira LS, Manente FA, et al. Dectin-1 expression by macrophages and related antifungal mechanisms in a murine model of Sporothrix schenckii sensu stricto systemic infection. Microb Pathog. 2017;110:78-84.

12. Snarr BD, Qureshi S, Sheppard D. Immune recognition of fungal polysaccharides. J Fungi. 2017;3:47.

13. Marcos CM, de Oliveira HC, de Melo WC, et al. Anti-immune strategies of pathogenic fungi. Front Cell Infect Microbiol. 2016;6:142.

14. Netea MG, Van der Graaf C, Van der Meer JWM, Kullberg BJ. Recognition of fungal pathogens by toll-like receptors. Eur J Clin Micobiol Infect Dis. 2004;23:672-676.

15. Wagener J, Malireddi RKS, Lenardon MD, et al. Fungal chitin dampens inflammation through IL-10 Induction mediated by NOD2 and TLR9 activation. PLoS Pathog. 2014;10:e1004050.

16. Stappers MHT, Clark AE, Aimanianda V, et al. Recognition of DHNmelanin by a C-type lectin receptor is required for immunity to Aspergillus. Nature. 2018;555:382-386.

17. Ene IV, Cheng SC, Netea MG, Brown AJ. Growth of Candida albicans cells on the physiologically relevant carbon source lactate affects their recognition and phagocytosis by immune cells. Infect Immun. 2013;81:238-248.

18. Rhome R, McQuiston T, Kechichian T, et al. Biosynthesis and immunogenicity of glucosylceramide in Cryptococcus neoformans and other human pathogens. Eukaryot Cell. 2007;6:1715-1726.

19. Deo SS, Gottlieb DJ. Adoptive T-cell therapy for fungal infections in haematology patients. Clin Transl Immunology. 2015;4:e40.

20. Della Terra PP, Rodrigues AM, Fernandes GF, Nishikaku AS, Burger E, de Camargo ZP. Exploring virulence and immunogenicity in the emerging pathogen Sporothrix brasiliensis. PLoS Negl Trop Dis. 2017;11:e0005903.

21. Wasylnka JA, Simmer MI, Moore MM. Differences in sialic acid density in pathogenic and non-pathogenic Aspergillus species. Microbiology. 2001;147(Pt 4):869-877.

22. Warwas ML, Watson JN, Bennet AJ, Moore MM. Structure and role of sialic acids on the surface of Aspergillus fumigatus conidiospores. Glycobiology. 2007;17:401-410.

23. Cassone A. Development of vaccines for Candida albicans: fighting a skilled transformer. Nat Rev Microbiol. 2013;11:884-891.

24. Torosantucci A, Bromuro C, Chiani P, et al. A novel glyco-conjugate vaccine against fungal pathogens. $J$ Exp Med. 2005;202:597-606.

25. Levy R, Segal E, Eylan E. Protective immunity against murine candidiasis elicited by Candida albicans ribosomal fractions. Infect Immun. 1981;31:874-878.

26. Han Y, Cutler JE. Antibody response that protects against disseminated candidiasis. Infect Immun. 1995;63:2714-2719.

27. Han Y, Ulrich MA, Cutler JE. Candida albicans Mannan Extract-Protein Conjugates Induce a Protective Immune Response against Experimental Candidiasis. J Infect Dis. 1999;179:1477-1484.

28. Liu M, Machova E, Nescakova Z, et al. Vaccination with mannan protects mice against systemic aspergillosis. Med Mycol. 2012;50(8): 818-828.
29. Sorgo AG, Brul S, de Koster CG, de Koning LJ, Klis FM. Iron restriction-induced adaptations in the wall proteome of Candida albicans. Microbiology. 2013;159(Pt 8):1673-1682.

30. Brad J. Spellberg ASI, Valentina Avanesian, et al. Efficacy of the antiCandida rAls3p-N or rAls1p-N vaccines against disseminated and mucosal candidiasis. J Infect Dis. 2006;194:256-260.

31. Schmidt CS, White CJ, Ibrahim AS, et al. NDV-3, a recombinant alumadjuvanted vaccine for Candida and Staphylococcus aureus, is safe and immunogenic in healthy adults. Vaccine. 2012;30:7594-7600.

32. Ibrahim AS, Luo G, Gebremariam T, et al. NDV-3 protects mice from vulvovaginal candidiasis through T- and B-cell immune response. Vaccine. 2013;31:5549-5556.

33. Coleman DA, Oh SH, Zhao X, et al. Monoclonal antibodies specific for Candida albicans Als3 that immunolabel fungal cells in vitro and in vivo and block adhesion to host surfaces. J Microbiol Methods. 2009; 78:71-78.

34. Moragues MD, Omaetxebarria MJ, Elguezabal N, et al. A monoclonal antibody directed against a Candida albicans cell wall mannoprotein exerts three anti-C. albicans activities. Infect Immun. 2003;71:5273-5279.

35. Brena S, Omaetxebarria MJ, Elguezabal N, Cabezas J, Moragues MD, Ponton J. Fungicidal monoclonal antibody C7 binds to Candida albicans Als3. Infect Immun. 2007;75:3680-3682.

36. Brena S, Cabezas-Olcoz J, Moragues MD, et al. Fungicidal monoclonal antibody $\mathrm{C} 7$ interferes with iron acquisition in Candida albicans. Antimicrob Agents Chemother. 2011;55:3156-3163.

37. Beucher B, Marot-Leblond A, Billaud-Nail S, Oh SH, Hoyer LL, Robert R. Recognition of Candida albicans Als3 by the germ tubespecific monoclonal antibody 3D9.3. FEMS Immunol Med Microbiol. 2009;55:314-323.

38. Torosantucci A, Chiani P, Bromuro C, et al. Protection by anti-betaglucan antibodies is associated with restricted beta-1,3 glucan binding specificity and inhibition of fungal growth and adherence. PLoS One. 2009; 4:e5392.

39. Laforce-Nesbitt SS, Sullivan MA, Hoyer LL, Bliss JM. Inhibition of Candida albicans adhesion by recombinant human antibody single-chain variable fragment specific for Als3p. FEMS Immunol Med Microbiol. 2008;54:195-202.

40. Clancy CJ, Nguyen ML, Cheng S, et al. Immunoglobulin G responses to a panel of Candida albicans antigens as accurate and early markers for the presence of systemic candidiasis. J Clin Microbiol. 2008;46: $1647-1654$.

41. Xin H, Dziadek S, Bundle DR, Cutler JE. Synthetic glycopeptide vaccines combining $\beta$ mannan and peptide epitopes induce protection against candidiasis. PNAS. 2008;105:13527-13531.

42. Bozza S, Clavaud C, Giovannini G, et al. Immune sensing of Aspergillus fumigatus proteins, glycolipids, and polysaccharides and the impact on Th immunity and vaccination. J Immunol. 2009;183:2407-2414.

43. Chaturvedi AK, Kavishwar A, Shiva Keshava GB, Shukla PK. Monoclonal immunoglobulin G1 directed against Aspergillus fumigatus cell wall glycoprotein protects against experimental murine aspergillosis. Clin Diagn Lab Immunol. 2005;12:1063-1068.

44. Ito JI, Lyons JM, Hong TB, et al. Vaccinations with recombinant variants of Aspergillus fumigatus allergen Asp $\mathrm{f} 3$ protect mice against invasive aspergillosis. Infect Immun. 2006;74:5075-5084.

45. Liu M, Capilla J, Johansen ME, et al. Saccharomyces as a vaccine against systemic aspergillosis: "the friend of man" a friend again? $J$ Med Microbiol. 2011;60:1423-1432.

46. Cenci E, Mencacci A, Bacci A, Bistoni F, Kurup VP, Romani L. T cell vaccination in mice with invasive pulmonary aspergillosis. J Immunol. 2000;165:381-388.

47. Rivera A, Van Epps HL, Hohl TM, Rizzuto G, Pamer EG. Distinct CD4+-T-cell responses to live and heat-inactivated Aspergillus fumigatus conidia. Infect Immun. 2005;73:7170-7179.

48. PerfectJR,CasadevallA.Cryptococcosis. InfectDis ClinNorthAm. 2002;16: $837-874$. 
49. Devi SJN. Preclinical efficacy of a glucuronoxylomannan-tetanus toxoid conjugate vaccine of Cryptococcus neoformans in a murine model. Vaccine. 1996;14:841-844.

50. Yuan R, Casadevall A, Oh J, Scharff MD. T cells cooperate with passive antibody to modify Cryptococcus neoformans infection in mice. Proc Natl Acad Sci. 1997;94:2483-2488.

51. Beenhouwer DO, Shapiro S, Feldmesser M, Casadevall A, Scharff MD. Both Th1 and Th2 cytokines affect the ability of monoclonal antibodies to protect mice against Cryptococcus neoformans. Infect Immun. 2001;69:6445-6455.

52. Yuan R, Casadevall A, Spira G, Scharff MD. Isotype switching from IgG3 to IgG1 converts a nonprotective murine antibody to Cryptococcus neoformans into a protective antibody. J Immunol. 1995;154:1810-1816.

53. Yuan RR, Spira G, Oh J, Paizi M, Casadevall A, Scharff MD. Isotype switching increases efficacy of antibody protection against Cryptococcus neoformans infection in mice. Infect Immun. 1998;66:1057-1062.

54. Rivera J, Casadevall A. Mouse genetic background is a major determinant of isotype-related differences for antibody-mediated protective efficacy against Cryptococcus neoformans. J Immunol. 2005;174:8017-8026.

55. Zhang H, Zhong Z, Pirofski LA. Peptide epitopes recognized by a human anti-cryptococcal glucuronoxylomannan antibody. Infect Immun. 1997;65:1158-1164.

56. Fleuridor R, Lees A, Pirofski L. A cryptococcal capsular polysaccharide mimotope prolongs the survival of mice with Cryptococcus neoformans infection. J Immunol. 2001;166:1087-1096.

57. Datta K, Lees A, Pirofski L. Therapeutic efficacy of a conjugate vaccine containing a peptide mimotope of cryptococcal capsular polysaccharide glucuronoxylomannan. Clin Vaccine Immunol. 2008;15:1176-1187.

58. Specht CA, Lee CK, Huang H, et al. Protection against experimental cryptococcosis following vaccination with glucan particles containing cryptococcus alkaline extracts. mBio. 2015;6:e01905-e01915.

59. Khan AA, Jabeen M, Chauhan A, Owais M. Vaccine potential of cytosolic proteins loaded fibrin microspheres of Cryptococcus neoformans in BALB/c mice. J Drug Target. 2012;20:453-466.

60. Wormley FL, Perfect JR, Steele C, Cox GM. Protection against cryptococcosis by using a murine gamma interferon-producing Cryptococcus neoformans strain. Infect Immun. 2007;75:1453-1462.

61. Wozniak KL, Young ML, Wormley FL. Protective immunity against experimental pulmonary cryptococcosis in T cell-depleted mice. Clin Vaccine Immunol. 2011;18:717-723.

62. Rosas ÁL, Nosanchuk JD, Casadevall A. Passive immunization with melanin-binding monoclonal antibodies prolongs survival of mice with lethal Cryptococcus neoformans infection. Infect Immun. 2001;69:3410-3412.

63. McClelland EE, Nicola AM, Prados-Rosales R, Casadevall A. Ab binding alters gene expression in Cryptococcus neoformans and directly modulates fungal metabolism. J Clin Invest. 2010;120:1355-1361.

64. Casadevall A, Cleare W, Feldmesser M, et al. Characterization of a murine monoclonal antibody to Cryptococcus neoformans polysaccharide that is a candidate for human therapeutic studies. Antimicrob Agents Chemother: 1998;42:1437-1446.

65. Bryan RA, Jiang Z, Howell RC, et al. Radioimmunotherapy is more effective than antifungal treatment in experimental cryptococcal infection. J Infect Dis. 2010;202:633-637.

66. Travassos LR, Taborda CP. Paracoccidioidomycosis vaccine. Hum Vaccin Immunother. 2012;8:1450-1453.

67. Puccia R, Schenkman S, Gorin PA, Travassos LR. Exocellular components of Paracoccidioides brasiliensis: identification of a specific antigen. Infect Immun. 1986;53:199-206.

68. Taborda CP, Juliano MA, Puccia R, Franco M, Travassos LR. Mapping of the T-cell epitope in the major 43-kilodalton glycoprotein of paracoccidioides brasiliensis which induces a Th-1 response protective against fungal infection in BALB/c mice. Infect Immun. 1998;66:786-793.

69. Marques AF, da Silva MB, Juliano MAP, Travassos LR, Taborda CP. Peptide immunization as an adjuvant to chemotherapy in mice challenged intratracheally with virulent yeast cells of Paracoccidioides brasiliensis. Antimicrob Agents Chemother. 2006;50:2814-2819.
70. Marques AF, da Silva MB, Juliano MAP, Munhõz JE, Travassos LR, Taborda CP. Additive effect of P10 immunization and chemotherapy in anergic mice challenged intratracheally with virulent yeasts of Paracoccidioides brasiliensis. Microbes Infect. 2008;10:1251-1258.

71. Pinto AR, Puccia R, Diniz SN, Franco MF, Travassos LR. DNA-based vaccination against murine paracoccidioidomycosis using the gp43 gene from Paracoccidioides brasiliensis. Vaccine. 2000;18:3050-3058.

72. Alegre ACP, Oliveira AF, Dos Reis Almeida FB, Roque-Barreira MC, Hanna ES. Recombinant paracoccin reproduces the biological properties of the native protein and induces protective Th1 immunity against Paracoccidioides brasiliensis infection. PLoS Negl Trop Dis. 2014;8:e2788.

73. Morais EA, Martins EM, Boelone JN, Gomes DA, Goes AM. Immunization with recombinant $\mathrm{Pb} 27$ protein reduces the levels of pulmonary fibrosis caused by the inflammatory response against Paracoccidioides brasiliensis. Mycopathologia. 2015;179:31-43.

74. Mora-Montes HM, Dantas Ada S, Trujillo-Esquivel E, de Souza Baptista AR, Lopes-Bezerra LM. Current progress in the biology of members of the Sporothrix schenckii complex following the genomic era. FEMS Yeast Res. 2015;15:fov065.

75. Rodrigues AM, Kubitschek-Barreira PH, Fernandes GF, de Almeida SR, Lopes-Bezerra LM, de Camargo ZP. Immunoproteomic analysis reveals a convergent humoral response signature in the Sporothrix schenckii complex. J Proteomics. 2015;115:8-22.

76. Medici NP, Del Poeta M. New insights on the development of fungal vaccines: from immunity to recent challenges. Mem Inst Oswaldo Cruz. 2015;110:966-973.

77. Rodrigues AM, Fernandes GF, Araujo LM, et al. Proteomics-based characterization of the humoral immune response in sporotrichosis: toward discovery of potential diagnostic and vaccine antigens. PLoS Negl Trop Dis. 2015;9:e0004016.

78. Alba-Fierro CA, Perez-Torres A, Toriello $\mathrm{C}$, et al. Immune response induced by an immunodominant $60 \mathrm{kDa}$ glycoprotein of the cell wall of Sporothrix schenckii in two mice strains with experimental sporotrichosis. J Immunol Res. 2016;2016:6525831.

79. Almeida SR. Therapeutic monoclonal antibody for sporotrichosis. Front Microbiol. 2012;3:409.

80. Nascimento RC, Espindola NM, Castro RA, et al. Passive immunization with monoclonal antibody against a 70-kDa putative adhesin of Sporothrix schenckii induces protection in murine sporotrichosis. Eur J Immunol. 2008;38:3080-3089.

81. de Lima-Franco D, Nascimento RC, Ferreira KS, Almeida SR. Antibodies against Sporothrix schenckii enhance TNF-alpha production and killing by macrophages. Scand J Immunol. 2011;75:142-146.

82. de Almeida JR, Kaihami GH, Jannuzzi GP, de Almeida SR. Therapeutic vaccine using a monoclonal antibody against a $70-\mathrm{kDa}$ glycoprotein in mice infected with highly virulent Sporothrix schenckii and Sporothrix brasiliensis. Med Mycol. 2015;53:42-50.

83. Portuondo DL, Batista-Duharte A, Ferreira LS, et al. A cell wall proteinbased vaccine candidate induce protective immune response against Sporothrix schenckii infection. Immunobiology. 2016;221:300-309.

84. Portuondo DL, Batista-Duharte A, Ferreira LS, et al. Comparative efficacy and toxicity of two vaccine candidates against Sporothrix schenckii using either montanide pet gel A or aluminum hydroxide adjuvants in mice. Vaccine. 2017;35:4430-4436.

85. Chen F, Jiang R, Wang Y, et al. Recombinant phage elicits protective immune response against systemic $\mathrm{S}$. globosa infection in mouse model. Sci Rep. 2017;7:42024.

86. Deepe GS, Wüthrich M, Klein BS. Progress in vaccination for histoplasmosis and blastomycosis: coping with cellular immunity. Med Mycol. 2005;43:381-389.

87. Wuthrich M, Filutowicz HI, Klein BS. Mutation of the WI-1 gene yields an attenuated Blastomyces dermatitidis strain that induces host resistance. J Clin Invest. 2000;106:1381-1389.

88. Gomez FJ, Gomez AM, Deepe GS. Protective efficacy of a 62-kilodalton antigen, HIS-62, from the cell wall and cell membrane of Histoplasma capsulatum yeast cells. Infect Immun. 1991;59:4459-4464. 
89. Gomez FJ, Allendoerfer R, Deepe GS. Vaccination with recombinant heat shock protein 60 from Histoplasma capsulatum protects mice against pulmonary histoplasmosis. Infect Immun. 1995;63:2587-2595.

90. Deepe GS. Prospects for the development of fungal vaccines. Clin Microbiol Rev. 1997;10:585-596.

91. Shubitz LF, Yu JJ, Hung CY, et al. Improved protection of mice against lethal respiratory infection with Coccidioides posadasii using two recombinant antigens expressed as a single protein. Vaccine. 2006;24:5904-5911.

92. Matthews RC, Burnie JP. Recombinant antibodies: a natural partner in combinatorial antifungal therapy. Vaccine. 2004;22:865-871.

93. Larsen RA, Pappas PG, Perfect J, et al. Phase I evaluation of the safety and pharmacokinetics of murine-derived anticryptococcal antibody $18 \mathrm{~B} 7$ in subjects with treated cryptococcal meningitis. Antimicrob Agents Chemother. 2005;49:952-958.
94. Nosanchuk JD, Steenbergen JN, Shi L, Deepe GS, Casadevall A. Antibodies to a cell surface histone-like protein protect against Histoplasma capsulatum. J Clin Invest. 2003;112:1164-1175.

95. Magliani W, Conti S, Salati A, et al. Therapeutic potential of yeast killer toxin-like antibodies and mimotopes. FEMS Yeast Res. 2004;5: 11-18.

96. Polonelli L, Morace G. Production and characterization of yeast killer toxin monoclonal antibodies. J Clin Microbiol. 1987;25:460-462.

97. Sears D, Schwartz BS. Candida auris: an emerging multidrug-resistant pathogen. Int J Infect Dis. 2017;63:95-98.

98. Liedke SC, Miranda DZ, Gomes KX, et al. Characterization of the antifungal functions of a WGA-Fc (IgG2a) fusion protein binding to cell wall chitin oligomers. Sci Rep. 2017;7:12187.
Infection and Drug Resistance

\section{Publish your work in this journal}

Infection and Drug Resistance is an international, peer-reviewed openaccess journal that focuses on the optimal treatment of infection (bacterial, fungal and viral) and the development and institution of preventive strategies to minimize the development and spread of resistance. The journal is specifically concerned with the epidemiology of antibiotic

\section{Dovepress}

resistance and the mechanisms of resistance development and diffusion in both hospitals and the community. The manuscript management system is completely online and includes a very quick and fair peerreview system, which is all easy to use. Visit http://www.dovepress.com/ testimonials.php to read real quotes from published authors. 